\title{
Is it time for BRCA1/2 mutation screening in the general adult population?: impact of population characteristics
}

\author{
Glenn E. Palomaki, PhD ${ }^{1}$
}

Two recent linked publications present perspectives on routine population-based screening for breast cancer using BRCA1/2 mutations in different populations. ${ }^{1,2}$ In the United States, an estimated 235,000 new breast cancer cases are diagnosed annually, resulting in more than 40,000 deaths. ${ }^{3}$ From an epidemiological viewpoint, the strongest factors associated with breast cancer are being female and advancing age. However, more than 60 years ago, it was clear that some families had higher than expected rates of breast and ovarian cancer; researchers theorized that this might be due to an inherited predisposition. A family history of breast/ovarian cancer or a personal history of early-onset cancer are strong risk factors for breast cancer, and systematic criteria for a positive family history have been established by professional organizations and/or government agencies (e.g., US Preventive Services Task Force, ${ }^{4}$ National Comprehensive Cancer Network ${ }^{5}$ ). Less strongly related risk factors include oral contraceptive use, weight, alcohol intake, and reproductive history. In 1994 and 1995, the tumor suppressor genes BRCA1 (ref. 6) and BRCA2 (ref. 7) were identified. Since then, the scientific community has extensively explored both the personal and population impact of carrying a deleterious mutation in these genes. Any new population-based screening test, such as testing for BRCA1 and BRCA2 mutations, requires consideration of key performance characteristics that evaluate both strengths and shortcomings before its introduction. ${ }^{8,9}$

The relationship between deleterious mutations in BRCA1/2 and breast cancer at the population level can be described by answering four epidemiologic questions: (i) What percentage of all women in the population carry a deleterious mutation? (ii) What percentage of women with breast cancer in the population carry one of these mutations? (iii) What percentage of women with a mutation develop breast cancer? (iv) What percentage of women will develop cancer by a given age, regardless of mutation status? Shorthand phrases for these four parameters are the carrier rate, the clinical sensitivity, the penetrance, and the population cumulative incidence, respectively. These four factors are interrelated; knowing three will allow the fourth to be computed. ${ }^{10}$ As an example, three founder mutations account for the majority of deleterious BRCA1/2 mutations in the Ashkenazi Jewish population, allowing an inexpensive molecular test to be used. A reasonably confident estimate of the carrier rate in this population is about 1 in 40 (2.5\%; range, 1 in 33 to 1 in 56). ${ }^{11}$ The proportion of all breast cancer cases attributable to these founder mutations (clinical sensitivity) is about $10 \%$ by age 70 (higher rates are found in younger women). The general cumulative incidence of breast cancer in the Ashkenazi population is also about $10 \%$ by age 70 (lower rates are found in younger women). Penetrance is more difficult to establish; published estimates (ranging from $30 \%$ to $70 \%$ ) are usually based on women with strongly positive family histories (index cases), and this may bias results toward overestimates. However, by using the previous three parameters, the expected penetrance of $40 \%$ can be computed as follows: (sensitivity $\times$ incidence $) /$ carrier $=$ penetrance, or $(10 \times 10) / 2.5=40$.

A recent publication by Gabai-Kapara et al. ${ }^{1}$ used a unique study design to better quantify penetrance estimates in the Ashkenazi Jewish population living in Israel. Rather than starting with women with an identified BRCA1/2 mutation (index cases), as in previous studies, they approached healthy Ashkenazi Jewish males for initial testing and documented a carrier rate of $2.1 \%$ ( 1 in 48 ). They then contacted and tested female relatives of the carrier males. Females with a mutation then were enrolled, along with a matched control group, and information about the family's history of breast/ovarian cancer was collected, which allowed a more reliable estimate of penetrance among female carriers to be determined. The general cumulative incidence of breast cancer by age 70 was $9.4 \%$, penetrance was $52 \%$, and clinical sensitivity was $11 \%$ (women with BRCA1/2 mutations accounted for $11 \%$ of all breast cancer cases). A penetrance of $49 \%$ can be estimated by using the carrier rate, clinical sensitivity, and population cumulative incidence; this is similar to the reported $52 \%$. The study is thus internally consistent, and all parameters also are externally consistent with those from other publications. The important difference in these findings is that they were derived from the general Ashkenazi Jewish population, rather than from followup with the families of index cases.

Given these findings, Gabai-Kapara et al. ${ }^{1}$ propose a pilot screening program in which BRCA1/2 founder mutation

${ }^{1}$ Division of Medical Screening and Special Testing, Department of Pathology and Laboratory Medicine, Women \& Infants Hospital, Alpert Medical School of Brown University, Providence, Rhode Island, USA. Correspondence: Glenn E. Palomaki (gpalomaki@ipmms.org) 
testing is offered to all younger women and genetic counseling and potential surgical interventions are offered to carriers. Figure 1a applies their findings to a hypothetical cohort of 100,000 Ashkenazi Jewish women aged 20 years. Founder mutation testing detects $11 \%$ of all breast cancers in the population ( $89 \%$ not detected); BRCA1/2 mutations are not associated with most cases of breast cancer. Two of every 92 women tested will carry a mutation, and one of these carriers will not develop breast cancer by age 70 (false-positive rate $1.1 \%$ ). If this model were to include ovarian cancer, the false-positive rate would be lower. Finally, the risk of breast cancer among those testing negative is $8.5 \%$. This relatively small reduction from the prior population risk of $9.4 \%$ is because most breast cancers in the general population are not associated with these mutations. Assuming a cost of testing for the three mutations of $\$ 200$, the cost per case detected (BRCA1/2 mutation carrier who will develop breast cancer by age 70 ) is about $\$ 19,000$. This cost is relatively low compared with, for example, prenatal screening for Down syndrome, in which each case detected may cost $\$ 200,000$ or more. This example also does not include the benefits of identifying increased risks of ovarian cancer, which would make such testing even more cost-effective.

Appreciating the call for a pilot program before routine implementation is important. Many implementation issues have not been fully explored, even though current family history protocols often contain a recommendation for immediate founder mutation testing if the woman is Ashkenazi Jewish. For example, will women who test negative understand that this result does not substantially reduce their lifetime risk of developing breast cancer (e.g., from 9.4 to $8.5 \%$ )? This modest risk reduction may seem surprising when viewed in the context of current clinical practice, which focuses on women with a positive family history (and a high prior risk). This practice

a

BRCA1/2 testing

in 20-year-old Ashkenazi Jewish women

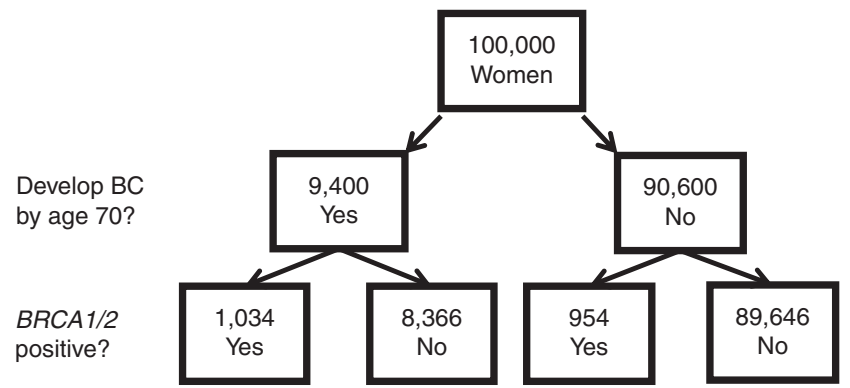

Carrier rate: $(1,034+954) / 100,000$

Clinical detection rate: $(1,034 / 9,400)$

Penetrance: $(1,034 /(1,034+954)$

General cum. incidence: $9,400 / 100,000$

$$
\begin{aligned}
& =2 \%(1 \text { in } 50) \\
& =11 \% \\
& =49 \% \\
& =9.4 \%
\end{aligned}
$$

contrasts with offering screening to the general population, in which women will have, on average, a much lower prior risk of breast cancer. Other questions also deserve consideration. Will women who test negative be more likely to avoid routine mammography and thus negatively affect current screening programs? Will the uptake of preventive surgeries among women who screen positive be considerably lower than current rates because many of these women will not have a positive family history? A carefully crafted programmatic approach to evaluate such screening in an appropriately sized target population would provide important information to aid decision making about routine testing. Funding agencies should look carefully at supporting such studies.

In a related publication, King and colleagues ${ }^{2}$ suggest that "it is time to offer genetic screening of these genes (BRCA1 and $B R C A 2$ ) to every woman, at about age 30 , in the course of routine medical care," but there is no call for a pilot phase. The setting is now the entire United States, where one can no longer test for three founder mutations but must instead use more complex and expensive techniques such as next-generation sequencing. Figure $1 \mathrm{~b}$ explores this further by asking the same epidemiologic questions as before. Some important numerical differences emerge (Figure 1b). The cumulative incidence of breast cancer in the general US population (9.4\%) and the penetrance of $B R C A 1 / 2$ mutations (52\%) can remain the same, but the carrier rate is about 10 times lower ( 1 in 400 , or $0.25 \%$ ). Because of this, only about $1.4 \%$ of all breast cancer cases are accounted for by women with one of these mutations (clinical sensitivity). The number of women identified with a mutation is now 254 in 100,000 , rather than 1,988 in 100,000 , as it is in the Ashkenazi Jewish population (Figure 1a). Given that mutation testing via next-generation sequencing will, of necessity, be more extensive (and expensive), the cost per case of cancer detected in a mutation

b

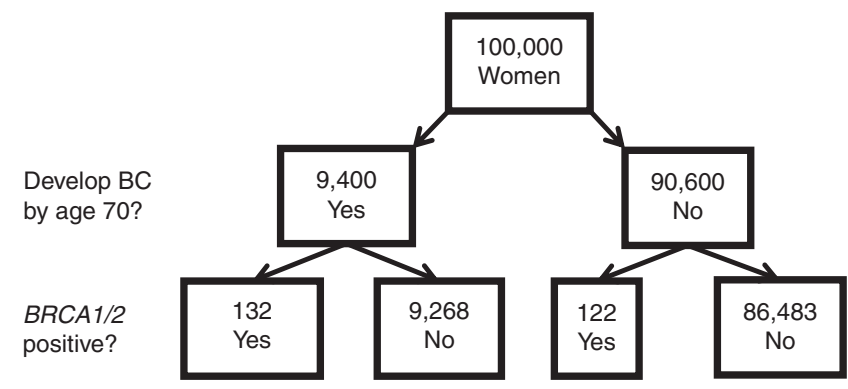

Figure 1 Flow diagram of a hypothetical cohort of 100,000 20-year-old women being tested for BRCA1/2 mutations to aid in avoiding breast cancer. The flowcharts show the application of four epidemiological parameters (shown below the figures) that allow the potential impact of screening for $B R C A 1 / 2$ to be determined in two distinct populations. (a) Testing in the Ashkenazi Jewish population; (b) the same program in the general US population, which differs by having a much lower mutation carrier rate and, subsequently, a lower clinical detection rate. See text for a more detailed description. 
carrier is considerably higher at about $\$ 760,000$ (assuming the cost of BRCA1/2 testing in this population is $\$ 1,000)$. Even if this cost were acceptable (or testing were less expensive), there are important issues, in addition to those described earlier, to address before routine implementation. Next-generation sequencing will identify variants of unknown clinical significance in at least $4 \%$ of women who test negative for deleterious mutations (about 4,000 in the hypothetical cohort of 100,000). King and colleagues suggest not reporting variants of unknown clinical significance as part of their proposed screening program. However, whether laboratories can withhold this information and whether providers and women opting for screening will be supportive of this approach has not yet been studied. Having information about where, how, and by whom such screening will be offered would also be valuable. For example, given that younger women are the target population, would screening involve obstetricians, primary-care providers, or both? A number of key questions designed for assessing this proposed population screening strategy can be answered by a pilot study. ${ }^{9}$ However, such a study will be both logistically difficult and expensive to conduct.

Together, these two publications offer an unusual opportunity to compare and contrast how distinct population differences, such as the mutation carrier rate, might influence the feasibility of population-based screening. In the Ashkenazi Jewish population, founder mutations are common and account for about $10 \%$ of all breast cancer cases; testing for these avoids any problems with variants of unknown clinical significance and is relatively inexpensive. Even so, pilot trials are indicated before launching widespread screening, to identify and resolve implementation issues. Such screening in the United States is considerably more complicated for several reasons, tilting the balance away from routine population screening, at least for the moment.

\section{DISCLOSURE}

The author declares no conflict of interest.

\section{REFERENCES}

1. Gabai-Kapara E, Lahad A, Kaufman B, et al. Population-based screening for breast and ovarian cancer risk due to BRCA1 and BRCA2. Proc Natl Acad Sci USA 2014;111:14205-14210.

2. King MC, Levy-Lahad E, Lahad A. Population-based screening for BRCA1 and BRCA2: 2014 Lasker Award. JAMA 2014;312:1091-1092.

3. American Cancer Society. Cancer Facts and Figures 2014. Atlanta. http://www cancer.org/acs/groups/content/@research/documents/webcontent/acspc042151.pdf. Accessed 2 October 2014.

4. US Preventive Services Task Force. Genetic risk assessment and BRCA mutation testing for breast and ovarian cancer susceptibility: recommendation statement. Ann Intern Med 2005;143:355-361.

5. NCCN Clinical Practice Guidelines in Oncology (NCCN Guidelines). Genetid Familial High-Risk Assessment: Breast and Ovarian. Version 2.2014. Jenkintown, PA, 2014.

6. Miki Y, Swensen J, Shattuck-Eidens D, et al. A strong candidate for the breast and ovarian cancer susceptibility gene BRCA1. Science 1994;266:66-71.

7. Wooster R, Bignell G, Lancaster J, et al. Identification of the breast cancer susceptibility gene BRCA2. Nature 1995;378:789-792.

8. Wald N, Cuckle H. Reporting the assessment of screening and diagnostic tests. Br J Obstet Gynaecol 1989;96:389-396.

9. Haddow JE, Palomaki GE. An introduction to assessing genomic screening and diagnostic tests. Nutr Today 2011;46:162-168.

10. McClain MR, Palomaki GE, Nathanson KL, Haddow JE. Adjusting the estimated proportion of breast cancer cases associated with BRCA1 and BRCA2 mutations: public health implications. Genet Med 2005;7:28-33.

11. MCClain MR, Nathanson KL, Palomaki GE, Haddow JE. An evaluation of BRCA1 and BRCA2 founder mutations penetrance estimates for breast cancer among Ashkenazi Jewish women. Genet Med 2005;7:34-39. 